\title{
FOUR-PROPELLERS UNDERWATER DRONE MODELLING
}

\author{
Mohamed MOUSTANIR ${ }^{{ }^{*}}$, Karim BENKIRANE ${ }^{2}$, Adil SAYOUTI ${ }^{1,2}$, Hicham MEDROMI ${ }^{1}$ \\ ${ }^{1}$ National Higher School of Electricity and Mechanic (ENSEM), Casablanca, Morocco, \\ ${ }^{2}$ Royal Navy School (ERN) of Morocco,
}

\begin{abstract}
We present in this presentation the design of an underwater robot with four propellers, without rudders or diving bars, using the resources of drones [1] for its evolution in the aquatic environment, the modeling is done in a perfect environment without forces or hydrodynamic couples.

Keywords: Mobile robot, underwater vehicle, Propellers, Modeling, Simulation, command thrusters, Propellers arrangement.
\end{abstract}

\section{Introduction}

The use of underwater vehicles, which was once an option, has become a necessity in many cases such as research, mapping or monitoring of sea depths. Also, the hostility of the underwater environment made these missions more difficult and more expensive, even impossible in certain cases. The use of these mobile robots offered a very advantageous alternative compared to Human intervention and often more to what the mission requires [1].

Architectural and control solutions generally optimized the choice of these machines. In this paper, we will focus first on the history of the main underwater devices and on the architecture of our machine, which will draw from the maneuverability of aerial drones with four rotors for its evolution in the marine environment; it will be the object of this work and also study the behavior of this robot with this type of propulsion by modeling and simulation [2].

These developments are part of the overall project initiated by the EAS team of the Computer Laboratory, systems and renewable energy (LISER) of the National School of Electrical and Mechanical (ENSEM) in Casablanca / Morocco.

\section{State of art}

Underwater vehicles have become increasingly present in many areas related to the seabed. They are used in both civilian and military sectors, starting with the oil industry and marine cartography and going through the rescue and mine search [3].

Several models and technologies of these machines appeared at their beginning remote controlled with the humane presence in the chain of decision, after * Corresponding author: moustanir@gmx.fr they created autonomous variants AUVs (Autonomous Underwater Vehicles) which filled the limitations of Remotely Operating Vehicles (ROVs) [4]. All these submersibles have been able to assert their importance and consolidate their position as an indispensable tool to avoid endangering humans and to reduce operating costs by automating some repetitive or reduced-decision tasks in relation to the fields aforementioned.

\section{Underwater vehicle classification}

"Underwater Vehicle" refers to any craft that can navigate below the surface of the water. There are several variations of these machines [5] and are classified according to several criteria depending on:

- Their energy or decision autonomies,

- Their range action or operational depth,

- Their dimensions or architectures,

- Their propulsion mode,

- The nature of the missions to be performed,

- Whether they are occupied by humans or not occupied.

In this part, we will present a classification according to the decisional autonomy.

\subsection{Human Operated Vehicle}

All type of vehicle whose human factor appears in the control chain [6], such as:

- HOS or HOV (Human Occupied Submersible / Vehicle): totally controlled by an on-board pilot, it canals contain more researchers. It is equipped with highdefinition cameras, projectors and robot arms to collect bottom samples and marine wildlife. Therefore, this category also includes military submarines. 
- ROV (Remotely Operated Vehicle). With no crew, it is totally remote controlled from the surface by a series of cables, it can have the same equipment as the HOS and can perform similar missions and reach greater depths.

-UUV (Untethered Underwater Vehicle):it is a gear operating without attachment to the surface, but there is an acoustic link between the surface and the submarine to allow control and data exchange.

\subsection{Autonomous Underwater Vehicle}

The Autonomous Underwater Vehicle (AUV) can operate completely without human control, which is important during long-term or repeated missions. They can, according to the implemented program, follow a race or interact with the environment to make their own decisions [7]; for example:

AUG (Autonomous Underwater Gliders): is distinguished by the ability to use ballasts for diving and wings to glide under the surface of the water for longterm missions (Fig. 1).

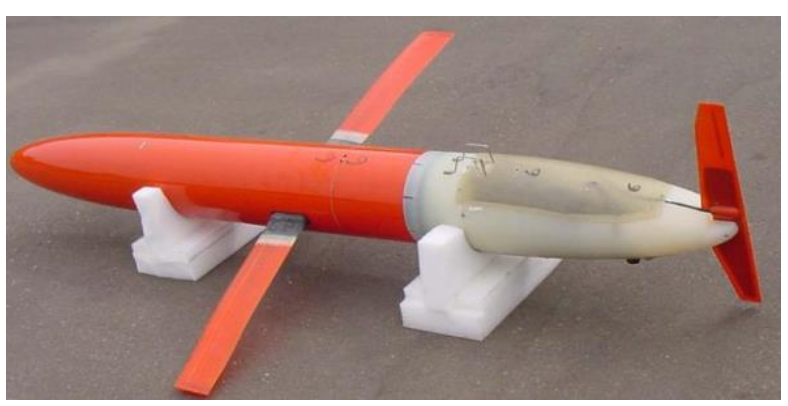

Figure 1: Spray Glider (BluefinRobotics)

BUVs (Biomimetic Underwater Vehicles): They are distinguished by their resemblance to living creatures that have been inspired for their way of moving.

Micro Autonomous Vehicles (MUVs): Avery important variant of AUVs, they find their applications in the military sector for recognition missions, antisubmarine warfare or mine search and can also be used to inspect submarine pipelines. [8].

\section{Model proposal}

\section{1 general concept}

The use of underwater machines or robots, which was once an option, has become a necessity in several fields such as research, cartography or seabed monitoring. Also, the hostility of the underwater environment made these missions more difficult and more expensive, even impossible in some cases. The use of these autonomous mobile robots offered an often very advantageous alternative more to what the mission requires compared to human intervention [1].

Architectural and control solutions generally optimized the choice of these machines. In this presentation we will demonstrate if a torpedo-shaped underwater robot can move in an aquatic environment without a rudder or a diving bar, with only the combination of thrusts from its thrusters (figure 1).

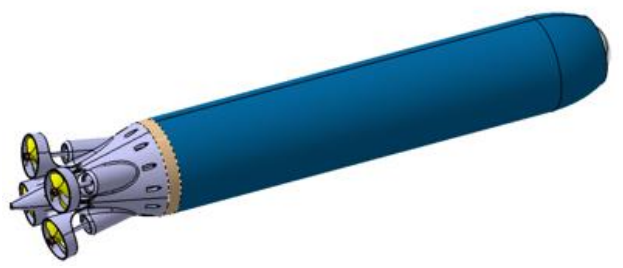

Figure 2 : Proposed model in perspective

The solution must take into account the following general conditions [2]:

- Use drone technologies and adapt them to this submersible,

- Like any classic submarine, it will need ballast for any static dive,

- At least three submerged propellers are required for dynamic diving,

- Eventually it will need a weight compensator installed at the rear to compensate for the weight of the front and thus balance the submersible.

\subsection{Architectural solution [2].}

\subsubsection{Advantages:}

- Solution already tested and functional;

- Wide ranges of hardware and software tools available for adaptation;

- Great maneuverability;

- Increased speed;

- Absence of rudders.

\subsubsection{Difficulties:}

- Very unfavorable environment (corrosion, short circuit, fluid resistance);

- Vertical rotor plane;

- Sensors not suitable for the aquatic environment;

- Weight distribution;

- Water tightness of the hull

\section{Robot modeling}

The modeling of the ROV (Remotely Operated Vehicle) includes the kinematic part that will focus on the movement and geometric relationships of the submarine. While the dynamic part will deal with the forces and torques acting on this machine.

\subsection{Model Kinematics}

The global position vector:

$$
\begin{gathered}
\eta=[x, y, z, \varphi, \theta, \psi]^{T} \\
\eta=\left(\eta_{1}, \eta_{2}\right)^{T} \quad \eta_{1}=(x, y, z)^{T} \quad \eta_{2}=(\varphi, \theta, \psi)^{T}
\end{gathered}
$$


In the same way we can combine the speeds:

$$
v=\left(v_{1}, v_{2}\right)^{T} \quad v_{1}=(u, v, w)^{T} \quad v_{2}=(p, q, r)^{T}
$$

In coordinate system $\mathrm{R}_{0}$ :

$$
\dot{\eta}_{1}=J_{C_{1}}\left(\eta_{2}\right) v_{1}
$$$$
\dot{\eta}_{2}=J_{C_{2}}\left(\eta_{2}\right) v_{2}
$$

with:

$$
\begin{gathered}
J_{C_{1}}\left(\eta_{2}\right)=\left(\begin{array}{ccc}
c \psi c \theta & -s \psi c \varphi+c \psi s \theta s \varphi & s \psi s \varphi+c \psi s \theta c \varphi \\
s \psi c \theta & c \psi c \varphi+s \psi s \theta s \varphi & -c \psi s \varphi+s \psi s \theta c \varphi \\
-s \theta & c \theta s \varphi & c \theta c \varphi
\end{array}\right) \\
J_{C_{2}}\left(\eta_{2}\right)=\left[\begin{array}{ccc}
1 & \sin \varphi \tan \theta & \cos \varphi \tan \theta \\
0 & \cos \varphi & -\sin \varphi \\
0 & \sin \varphi / \cos \theta & \cos \varphi / \cos \theta
\end{array}\right]
\end{gathered}
$$

Euler's angles (figure 2) correspond in robotics to the system commonly called R.T.L which designates the Roll, Pitch and $\operatorname{Yaw}(\varphi, \theta$ and $\psi)[8]$ :

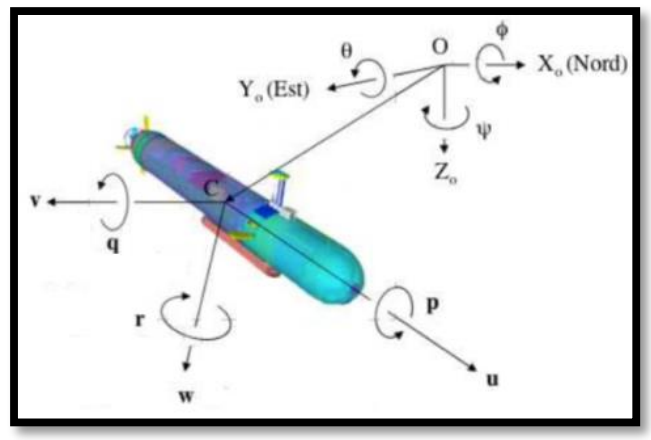

Figure 3 : Representation of Euler angles [3]

\subsection{Model dynamics}

In general, we can consider that the $\mathrm{R}_{0}$ coordinate system is a Galilean coordinate system except that it is fixed to the surface of the Earth. Thus, the forces generated by the rotation of the earth on the vehicle can be neglected compared to the hydrodynamic forces.

The dynamic simulation will be inspired by the work of Fossen [7].

The fundamental principle of the dynamics applied to the mobile in this reference gives [4].:

$$
M \dot{v}=f_{c}+f_{g}+f_{h}+\tau+w
$$

Where the vectors:

- $\quad \dot{v}=[\dot{u}, \dot{v}, \dot{w}, \dot{p}, \dot{q}, \dot{r}]^{T}$ : vehicle accelerations;

- $f_{c}$ : forces and torques of drive inertia and Coriolis;

- $f_{g}$ : forces and torques induced by weight and Archimedes' thrust;

- $f_{h}$ : hydrodynamic forces and torques;

- $\tau$ : forces and torques produced by actuators;

- $\quad w$ : external disturbances (waves ...).

\subsubsection{Inertia et Coriolis}

As the robot moves at low speeds, the forces and torques of drive and Coriolis inertia can be neglected

\subsection{2 weight and Archimedes' thrust}

$$
\begin{aligned}
& W=m \cdot g \\
& B=\rho \cdot \nabla \cdot g
\end{aligned}
$$

The forces that the submarine undergoes in water are Archimedes' push and that of its own weight and are written

with:

- $m$ : vehicle mass.

- $g$ : earth acceleration.

- $\rho:$ water density.

- $\quad \nabla:$ displaced water volume.

Thus, the vector of hydrostatic forces can be written as [1]:

$$
f_{g}=\left[\begin{array}{c}
-(W-B) \sin \theta \\
(W-B) \cos \theta \sin \varphi \\
(W-B) \cos \theta \cos \varphi \\
\left(y_{g} W-y_{b} B\right) \cos \theta \cos \varphi-\left(z_{g} W-z_{b} B\right) \cos \theta \sin \varphi \\
-\left(x_{g} W-x_{b} B\right) \cos \theta \cos \varphi-\left(z_{g} W-z_{b} B\right) \sin \theta \\
\left(x_{g} W-x_{b} B\right) \cos \theta \cos \varphi-\left(y_{g} W-y_{b} B\right) \sin \theta
\end{array}\right]
$$

with:

- B: Archimedes' thrust (Buoyancy) in N.

- W: Weight in N.

- $\left(x_{g}, x_{g}, x_{g}\right)$ : the position of the center of gravity in $\mathrm{Rv}$.

- $\left(x_{b}, x_{b}, x_{b}\right)$ : the position of the center of gravity in Rv.

If we consider the center of thrust and the center of the reference $\mathrm{Rv}$, is on the same axis $(\mathrm{Oz})$, and that the center of gravity must be below the center of thrust for the submarine to keep its position of initial equilibrium under the effect of the return torque (Figure 4) :

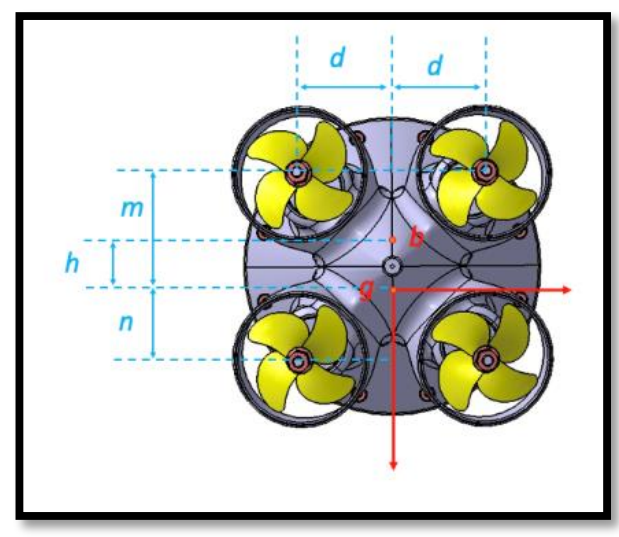

Figure 4: "x" Configuration in rear view. 
From where $x_{b}=0, y_{b}=0$ and $z_{b}=-h$, and that the mass of the submarine is distributed symmetrically with respect to the three planes $(x z),(x y)$ and $(y z)$, we know that in $\mathrm{R}_{\mathrm{V}} x_{g}=0, y_{g}=$ 0 et $z_{g}=0$, the same at equilibrium $\mathrm{W}=\mathrm{B}$ :

$$
f_{g}=\left[\begin{array}{c}
0 \\
0 \\
0 \\
W h \cos \theta \sin \varphi \\
W h \sin \theta \\
0
\end{array}\right]
$$

\subsubsection{Hydrodynamic forces and torques}

In this first study we will momentarily start the simulation without taking into account the hydrodynamic forces and torques, these will be presented in detail in the next publication.

\subsubsection{External disturbances}

Considering that the submarine will evolve in calm waters without the presence of waves or currents, these disturbances can be neglected.

\subsubsection{Actuator effects}

They generally refer to any source of thrust exerted on the craft. We will limit ourselves in this study to the propeller thrusters which are installed on the craft and which will be driven by electric motors.

Modeling the thrust of a thruster is relatively tricky because it depends on several parameters and is complicated by the coupling of many thrusters [4].

In our case, the four thrusters are directed along the $\mathrm{Ox}$ axis, depending on the configuration of the thrusters seen from the rear (Figure 19):

The vector of forces and torques applied to the vehicle by the actuators is generally defined as follows:

$$
\tau=\left(f_{x}, f_{y}, f_{z}, \Gamma_{x}, \Gamma_{y}, \Gamma_{z}\right)^{T}
$$

In our case, since we have four thrusters directed along the Ox axis, then:

$$
\tau=\left(f_{x}, 0,0,0, \Gamma_{y}, \Gamma_{z}\right)^{T}
$$

Hence the matrix of actuators:

$$
\tau=T . f_{i}
$$

Who becomes:

$$
\tau=\left[\begin{array}{cccc}
1 & 1 & 1 & 1 \\
0 & 0 & 0 & 0 \\
0 & 0 & 0 & 0 \\
0 & 0 & 0 & 0 \\
-d-h / 2 & -d-h / 2 & +d-h / 2 & +d-h / 2 \\
+d & -d & -d & +d
\end{array}\right] \cdot\left[\begin{array}{l}
f_{1} \\
f_{2} \\
f_{3} \\
f_{4}
\end{array}\right](15)
$$

with:

Applied to the studied ROV (figure 3):

$$
\tau=\left(f_{x}, 0,0,0, \Gamma_{y}, \Gamma_{z}\right)^{T}
$$

\subsection{Simulation}

For the simulation, we will implement the program in the Matlab language. The course of the trajectory will be visualized on a 3D interface. The program will integrate the ROV's own parameters as well as the equations which govern its movement to visualize the vehicle simulation [5].

The ROV equation of state system can be defined as:

$$
\left\{\begin{array}{c}
\dot{\eta}(t)=J_{C}(\eta) \cdot v(t) \\
\dot{v}(t)=M^{-1}\left(T \cdot f_{i}-W(\eta)-D(v) \cdot v(t)\right)
\end{array}\right.
$$

But for this first simulation in an ideal environment, it can be defined as follows:

$$
\left\{\begin{array}{l}
\dot{\eta}(t)=J_{C}(\eta) \cdot v(t) \\
\dot{v}(t)=M^{-1}\left(T \cdot f_{i}\right)
\end{array}\right.
$$

with:

$$
J_{C}=\left[\begin{array}{llllll}
J_{C_{1}}\left(\eta_{2}\right) & 0 & 0 & 0 \\
0 & 0 & 0 & 0 & 0 & 0 \\
0 & 0 & 0 & J_{C_{2}}\left(\eta_{2}\right) \\
0 & 0 & 0 &
\end{array}\right]
$$

and:

$M=-\left[\begin{array}{cccccc}X_{\dot{u}}+m & 0 & 0 & 0 & 0 & 0 \\ 0 & Y_{\dot{v}}+m & 0 & 0 & 0 & 0 \\ 0 & 0 & Z_{\dot{w}}+m & 0 & 0 & 0 \\ 0 & 0 & 0 & K_{\dot{p}}+I_{x x} & 0 & 0 \\ 0 & 0 & 0 & 0 & M_{\dot{q}}+I_{y y} & 0 \\ 0 & 0 & 0 & 0 & 0 & N_{\dot{r}}+I_{z z}\end{array}\right]$

In this part, several thruster power configurations will be simulated to see the general dynamic behavior of the underwater robot.

Some numerical data specific to the ROV:

$$
\begin{aligned}
J_{\Delta y}=J_{\Delta z}= & 1 / 2 \\
J_{\Delta x} & =0,143 \mathrm{Kg} \cdot \mathrm{m}^{2}=3,504 \mathrm{Kg} \cdot \mathrm{m}^{2} \\
r_{1} & =60 \mathrm{~mm} \\
r_{2} & =60 \mathrm{~mm} \\
l & =1,2 \mathrm{~m} \\
m & =28,6 \mathrm{Kg} \\
d & =90 \mathrm{~mm} \\
h & =15 \mathrm{~mm}
\end{aligned}
$$




\section{Numerical resolution}

These equations can be solved numerically by several methods of solving differential equations, nevertheless we will use in this chapter the 4th order Runge-Kutta method [9].

\subsection{Implementation}

This method is a general method usable at any order of differential equations. It suffices then to reduce the order ' $n$ ' of the equation to be solved to ' $n$ ' equations to solve. It is more complex and involves four times more calculations than the Euler method (for a first order differential equation for example), but it is much more reliable. The numerical diagram of this resolution for a 1 st order differential equation is written in the form with the 4th order Runge-Kutta method [9]:

$$
\begin{gathered}
y_{(t=0)}=y_{0} \\
K_{1}=f\left(t_{0}, y_{0}\right) \cdot d t \\
K_{2}=f\left(t_{0}+\frac{d t}{2}, y_{0}+\frac{K_{1}}{2}\right) \cdot d t \\
K_{3}=f\left(t_{0}+\frac{d t}{2}, y_{0}+\frac{K_{2}}{2}\right) \cdot d t \\
K_{4}=f\left(t_{0}+d t, y_{0}+K_{3}\right) \cdot d t \\
y_{1}=y_{0}+\frac{1}{6} \cdot\left(K_{1}+2 K_{2}+2 K_{3}+K_{4}\right)
\end{gathered}
$$

In our case, we will still need a 4th order resolution by this method. However, not for a 1st order differential equation, but this time for the 2 nd order. For that, we will decompose this equation into a system of 2 equations of the 1st order and the numerical diagram in this case will be analogous, but we will solve the 2 equations in parallel at the same time:

The previously described algorithm is implemented as a program in Matlab, the results will be presented according to some of the most common thruster configurations

\subsection{Results}

we are going to take several cases of combinations of the powers of the thrusters and we will see how the robot behaves in each case.

To give a quantitative idea of the thrust developed by the thrusters we will indicate the values assigned to each thruster in the order [F1, F2, F3, F4].

the proportionality between the horizontal thrusters is defined by: $p=\left(1+\frac{h}{n}\right)$.

Exemple : $F_{1}=14, F_{4}=22$,
- Thrusters have proportionally the same thrust $[14,14,22,22]$ :

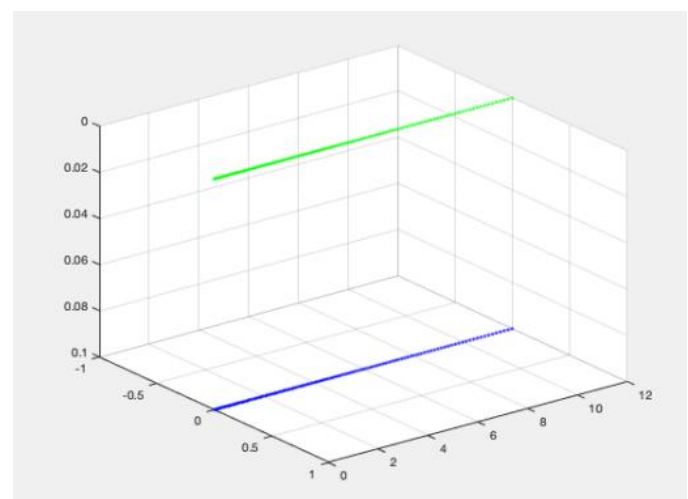

Figure 5: Thrusters have proportionally the same thrust.

- Vertical thrusters have proportionally the same thrust $[14,21,33,22]$ :

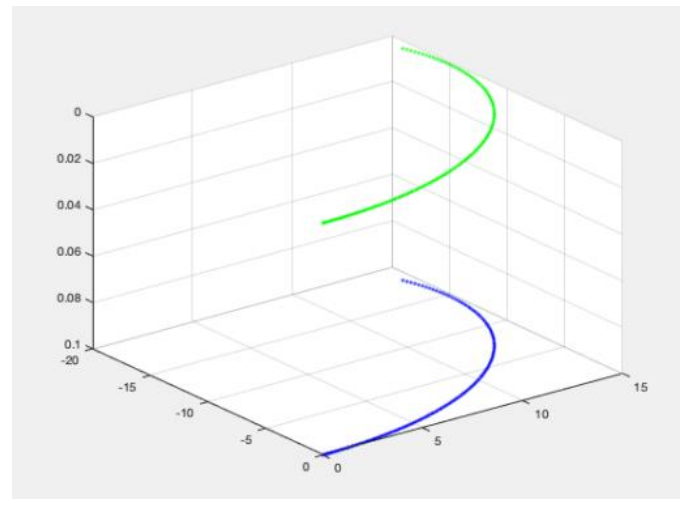

Figure 6: Vertical thrusters have proportionally the same thrust.

- Horizontal thrusters have the same thrust $[20,20,10,10]$ :

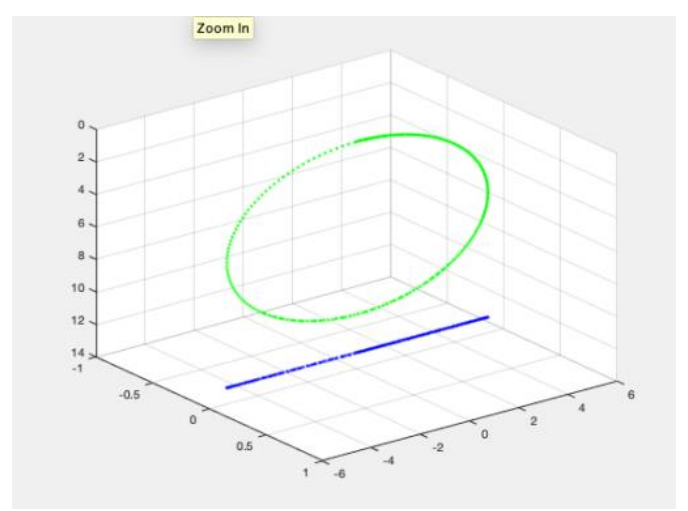

Figure 7: Horizontal thrusters have the same thrust.

\section{Realization}

For reasons of time savings, the production part has also started, prototyping and 3D printing are at a very advanced stage. 


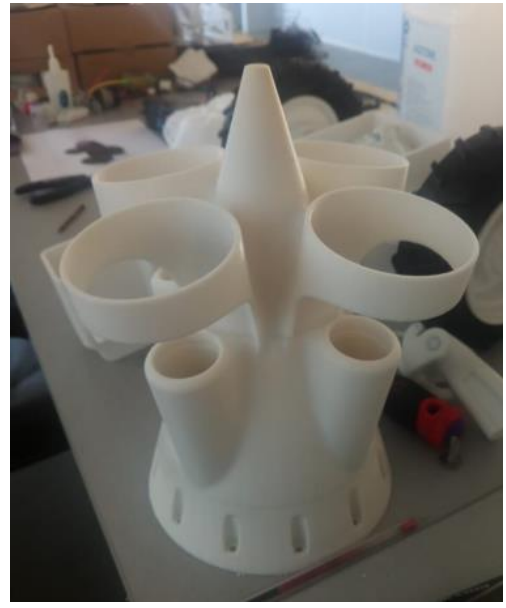

Figure 8: ROV's stern in 3D prototyping

The control and wiring diagrams are in progress.

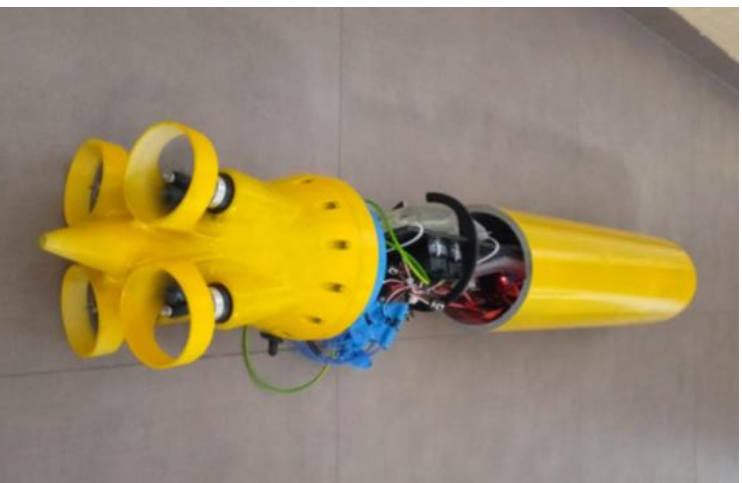

Figure 9: ROV's assembly in progress

In these figures (figure 8-9), we have seen how the ROV is partly carried out, the assembly and the wiring are in an advanced stage, remain the dry tests, to then begin those in real conditions.

\section{Conclusion}

In this publication we were able to show theoretically that a marine robot with four thrusters can move in the marine environment without having to use rudders or diving bars.

The various thrust combinations of the thrusters (figures 5-6-7) and the most common ones are tested and we have achieved the expected results.

In the next publication we will discuss the rest of the modeling and simulation of the ROV in a real environment with the introduction of gravity, hydrodynamic forces and torques.

\section{References:}

Russell B. Wynn, Veerle A.I. Huvenne, Timothy 1. P. Le Bas, Bramley J. Murton, Douglas P Connelly, Brian J. Bett a, Henry A. Ruhl, Kirsty J. Morris, J. Peakall, Daniel R. Parsons, Esther J. Sumner, Stephen E. Darby, Robert M. Dorrell, James E. Hunt, Autonomous Underwater Vehicles (AUVs): Their past, present and future

contributions to the advancement of marine geoscience, Marine Geology, Vol. 352, 2014

2.

A. Khadhraoui, 'Modélisation et simulation interactive pour la navigation d'un robot sousmarin de type ROV Observer" Ph.D. Thesis, Dept. Sciences et technologies de l'information et de la communication, Université Paris-Saclay, Paris, France, 2015.

M. Moustanir, K. Benkirane, A. Sayouti, H.

3. Medromi, Submarine Drone, First Edition of the Doctoral Days on Engineering Science ENSEM, Hassan II University of Casablanca May 2018

V. Creuze, B. Jouvencel, Seabed perception and 4. following for autonomous underwater vehicles, Traitement du Signal, Vol. 20, n. 4, 2003.

V. Creuze, Robots marins et sous-marins Perception, modélisation, commande, Techniques de l'Ingénieur, 2014.

M. Moustanir, K. Benkirane, A. Sayouti, H. Medromi, Four Propellers Architecture Proposed for the Submarine Drone, International Research Journal of Engineering and Technology (IRJET) Volume 6, Issue 11, November 2019

7. L. Jaulin, La robotique mobile, ISTE editions Ltd, Great London Britain, 2015.

T.I. Fossen, Guidance and Control of Ocean 8. Vehicles, John Wiley and Sons, Inc., New York (1994).

Society of Naval Architects and Marine 9. Engineers, "Nomenclature for treatingthe motion of a submerged body through a fluid," Tech. Rep., 1950

M. Crouzeix, A.L. Mignot: Analyse Numérique 10. des Équations Différentielles. Masson.

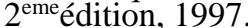

X. Liang, Y. Pang, L. Wan and B. Wang, 11. Dynamic Modelling and Motion Control for Underwater Vehicles with Fins, Alexander V. Inzartsev's Book, Underwater Vehicles, 2008, ITech, Vienna, Austria.

M. Schwing, Résolution numérique d'une equation différentielle, Méthode de RUNGEKUTTA RK4, IUFM de Lorraine

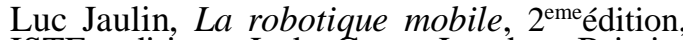

13. ISTE editions Ltd, Great London Britain, 2015 .

Rui Yang, Modeling and robust control 14. approach for autonomous underwater vehicles, Automatic, Université de Bretagne occidentale NNT: 2016BRES0011, Brest, France, 2016.

M. Crouzeix, A.L. Mignot: Analyse Numérique des Équations Différentielles. Masson.

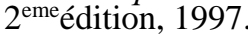

J. Geandrot, Méthodes numériques: Méthodes d'Euler de deRunge-Kuttad'ordre4 pour des équations du premier ordre ou du deuxième, 2003. 